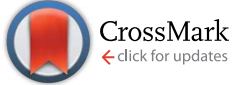

Cite this: RSC Adv., 2015, 5, 8895

Received 29th September 2014 Accepted 15th December 2014

DOI: 10.1039/c4ra11470a

www.rsc.org/advances

\section{Structure determination of molecular nanocomposites by combining pair distribution function analysis and solid-state NMR $\uparrow$}

\author{
E.-E. Bendeif, ${ }^{\star a b}$ A. Gansmuller, ${ }^{a b}$ K.-Y. Hsieh, ${ }^{\text {ab }}$ S. Pillet, ${ }^{\text {ab }}$ Th. Woike, ${ }^{c}$ M. Zobel, ${ }^{d}$

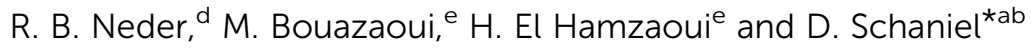

Transparent mesoporous silica monoliths of well-controlled porosity and a narrow pore size distribution around $6 \mathrm{~nm}$ have been used to prepare sodium nitroprusside (SNP) nanocomposites. The obtained nanomaterials could be characterised using X-ray total scattering coupled to atomic pair distribution function analysis (PDF) and solid-state NMR spectroscopy. The PDF analysis allows for a structural description of the confined species, as well as for the identification of the various coexisting phases: SNP isolated molecules and SNP crystalline nanoparticles. The model obtained suggests that the nanocrystals have the same molecular structure as the bulk crystalline material and measure about $6 \mathrm{~nm}$ in diameter. This result is quite exceptional because the space available inside the pores is only about ten times the size of the molecules. The multi-nuclei solid state NMR investigation confirms the structural model proposed by the PDF analysis and assigns the isolated molecules to the dynamic disorder of a solvated phase. The latter approach additionally provides quantitative information on the relative ratio between the dynamic molecules and the rigid nanocrystals. This result is exploited to study the evolution of the two confined SNP phases with respect to solvating water molecules. We show that the confined SNP nanocrystals can be easily dissolved when storing the nanocomposites at increasing atmospheric relative humidity.

\section{Introduction}

Porous silica gels have excellent biocompatibility, are easy to prepare, are transparent over a wide spectral range, and their pore size (1-100 $\mathrm{nm}$ ) can be adjusted for the targeted incorporation of nanoparticles or molecules. ${ }^{\mathbf{1 , 2}}$ Moreover, silica gels can themselves be prepared as nanoparticles with pore sizes in the nanometer range, and with appropriate surface coatings for targeting and entering different types of cells, ${ }^{3}$ as well as sensitive to a variety of triggers (photochemical, photothermal, enzymatic, etc.). ${ }^{4}$ These properties make porous silica composites ideal host materials for Active Pharmaceutical Ingredients (APIs), such as nitric oxide (NO) donors. ${ }^{5}$

\footnotetext{
'Université de Lorraine, CRM2, UMR 7036, Vandoeuvre-les-Nancy, F-54506, France. E-mail: el-eulmi.bendeif@univ-lorraine.fr; dominik.schaniel@univ-lorraine.fr

${ }^{b}$ CNRS, CRM2, UMR 7036, Vandoeuvre-les-Nancy, F-54506, France

${ }^{c}$ Institut für Strukturphysik, TU Dresden, Zellescher Weg 16, Dresden, Germany ${ }^{d}$ Crystallography and Structural Physics, University of Erlangen-Nürnberg, Staudtstr. 3, D-91058 Erlangen, Germany

${ }^{e}$ Laboratoire de Physique des Lasers, Atomes et Molécules (PhLAM), CNRS (UMR 8523), IRCICA (CNRS, USR 3380), Université Lille 1-Sciences et Technologies, Bâtiment P5, Villeneuve d'Ascq, F-59655, Cedex, France

$\uparrow$ Electronic supplementary information (ESI) available. See DOI: 10.1039/c4ra11470a
}

In order to be useful as API-carrying materials, one must assure that the pharmaceutical activity - or on more general grounds, the desired property - of the functional guest molecule is maintained after encapsulation in the porous host network. Some important parameters that may influence the activity are the size of the nanoparticle and its solubility, as well as the surrounding $\mathrm{pH}$-value and the relative humidity. The release kinetics and, therefore, the bioavailability of an API will, for example, strongly depend on the ability of the nanoparticles to dissolve in the pores and the ability of the solvated molecules to leave the porous host network. Furthermore, the host material could affect the structural integrity of the active ingredient or adsorb it on its inner surface, thus inhibiting the desired application. As has been shown for 'simple'6 (e.g. benzene) and more complex ${ }^{7}$ (e.g. isobutyric acid) liquids, the phase transition temperatures can be modified when the liquid is entrapped inside a porous matrix. This so-called confinement effect has also been observed for encapsulated organic solids, including anti-inflammatory APIs, ${ }^{8-10}$ where the particle structure and even the physical state of the molecules are different to those in the bulk material. The confinement effect on melting and glass transition temperatures mainly depends on the pore size and the relative strength between the guest/guest and host/guest interactions. ${ }^{\mathbf{1 1}, \mathbf{1 2}}$ For weak host/guest interactions, these temperatures are typically lower than for the bulk material. 
When the pore size is reduced, the decrease in the melting temperature becomes even greater, in qualitative agreement with the Gibbs-Thomson equation. ${ }^{\mathbf{1 3 , 1 4}}$ According to a study combining Dielectric Relaxation Spectroscopy and Differential Scanning Calorimetry experiments with Grand Canonical Monte Carlo simulations, ${ }^{15}$ fluids confined in cylindrical nanopores (e.g. MCM41) should freeze into a single crystalline structure for average pore diameters larger than $20 \sigma$ ( $\sigma$ being the diameter of the guest molecule). However, fluids or mixtures $^{\mathbf{1 6 , 1 7}}$ in pores with a diameter between $15 \sigma$ and $20 \sigma$ should essentially crystallise into a frustrated crystal structure, with the rest forming an amorphous or liquid region. Finally, for pore sizes smaller than $15 \sigma$, even partial crystallisation should not occur. These boundaries can however be modulated by the composition and the roughness of the pore surface.

It is thus desirable to utilise experimental methods that allow for a detailed structural characterisation of the functional guest and its interaction with the host, especially as a function of external parameters like temperature and environmental humidity. We have recently shown that the structure of inorganic complexes, those isolated inside $1 \mathrm{~nm}$ pores, can be analysed by total scattering methods and by subsequent modelling with the pair distribution function (PDF). We additionally demonstrated that, in combination with nuclear magnetic resonance (NMR), the structure, the dynamics, and the interaction of the isolated solvated molecules with their porous hosts could be characterised. ${ }^{18}$ We show here for $\mathrm{Na}_{2}\left[\mathrm{Fe}(\mathrm{CN})_{5} \mathrm{NO}\right] \cdot 2 \mathrm{H}_{2} \mathrm{O}$, sodium nitroprusside (SNP) that crystalline nanoparticles can be grown inside an amorphous silica matrix with a pore diameter as small as $6 \mathrm{~nm}$. We also demonstrate that the structure inside the matrix is heterogeneous and that the ratio between the nanoparticles and solvated molecules can be determined by combining PDFanalysis and NMR spectroscopy as a function of the atmospheric relative humidity.

SNP is an API used for the regulation of blood pressure ${ }^{19}$ and is attracting attention due to its potential use in a variety of other medical applications, due to its possibility to release the NO-ligand. ${ }^{20}$ Furthermore, SNP has photochromic and photorefractive properties, also rendering the material interesting for optical applications, such as data storage or all-optical switching. ${ }^{21,22}$ We have already studied SNP incorporated into silica matrices with pore sizes of $1 \mathrm{~nm}$ by combining PDF and NMR. ${ }^{18}$ The detailed structural analysis using X-ray total scattering and PDF-analysis clearly showed that SNP occurs as isolated solvated complexes, thus maintaining its structural integrity and only changing the cation-anion arrangement with respect to the crystalline phase. Thereby, the $\mathrm{Na}^{+}$cations arrange themselves in the equatorial plane of the $\left[\mathrm{Fe}(\mathrm{CN})_{5} \mathrm{NO}\right]^{2-}$ anion, cis to the NO. Since the SNP complexes in this case are isolated from each other, the PDF spectrum exhibits mainly intramolecular distances, and thus this can serve as an unambiguous fingerprint of the encapsulated molecular complex. These results were further confirmed by a multinuclear NMR study. NMR is especially suited, due to its local character and its sensitivity towards the ${ }^{23} \mathrm{Na},{ }^{13} \mathrm{C},{ }^{1} \mathrm{H}$ and ${ }^{29} \mathrm{Si}$ isotopes present in the studied material. From the ${ }^{29} \mathrm{Si}$ spectra, we could infer that the host matrix was not changed by the incorporation of the guest, while the ${ }^{23} \mathrm{Na}$ spectra confirmed the absence of a crystalline SNP phase. The ${ }^{1} \mathrm{H}$ spectra, on the other hand, showed that there is always a certain amount of water molecules present inside the porous network, which it is difficult to completely remove. Finally, the ${ }^{13} \mathrm{C}$ spectra showed evidence of isotropic rotational diffusion of the solvated SNP complexes inside the matrix pores. Therefore, the $\mathrm{H}_{2} \mathrm{O}$ present in the pores could not be reasonably modelled by the PDF analysis.

If the size of the pores in the network is increased, e.g. in order to enhance the drug releasing capabilities or drug concentration, the SNP complexes can aggregate or even crystallise in the form of nanoparticles. In this case, important questions are raised as to how much of the active ingredient occurs in the crystalline form, and how can this amount be controlled. Consequently, in order to study these questions and to extend our characterisation approach, we prepared silica xerogels with pore sizes of $6 \mathrm{~nm}$ and loaded them with SNP (from aqueous solution). Herein, we demonstrate using PDF and NMR that crystalline SNP nanoparticles can be grown inside pores of $6 \mathrm{~nm}$. We were able to observe that both solvated molecules and nanoparticles of about $6 \mathrm{~nm}$ diameter were incorporated and grown inside the matrix. NMR detected again the presence of water, the amount depending on the environmental humidity. From the ${ }^{23} \mathrm{Na}$ and ${ }^{13} \mathrm{C}$ spectral signatures, an unambiguous assignment of the liquid and solid SNP phases could be made, and the corresponding ratio could then be determined.

\section{Experimental}

\subsection{Synthesis of mesoporous silica monoliths}

The monolithic porous silica matrices hosts were synthesised according to the procedure previously reported. ${ }^{23-25}$ The transparent mesoporous monoliths were obtained by hydrolysis and polycondensation of TMOS, followed by a drying step, and then annealing in air at $850{ }^{\circ} \mathrm{C}$. This sol-gel process yields a large range of mesoporous xerogels with well-controlled pore sizes.

\subsection{Preparation of SNP-silica nanocomposites}

$\mathrm{SNP} @ \mathrm{SiO}_{2}$ nanocomposites were obtained following the wet impregnation procedure we published earlier. ${ }^{18}$ The prepared empty monoliths were loaded in an aqueous solution of SNP salt $\left(c=3.507 \mathrm{~mol} \mathrm{~L}^{-1}\right)$. The silica matrices were slowly added into the SNP solution in order to prevent any cracking. The monoliths, initially transparent, then became progressively more and more brown-orange, indicating diffusion of the SNP complexes into the silica pore network. After about 24 hours, the monoliths displayed a homogeneous colouring, and were then kept at $50{ }^{\circ} \mathrm{C}$ for 7 days in an oven. A final drying step was performed at room temperature in desiccators coupled with a primary pump ( $c a \cdot 10^{-2}$ mbar) for about 3 days, in order to remove the remaining solvent. A cross-section of the doped monolith exhibited a uniform brown-orange colour, confirming the homogeneous distribution of the SNP complexes. Fig. 1 shows the empty and the doped monoliths. 

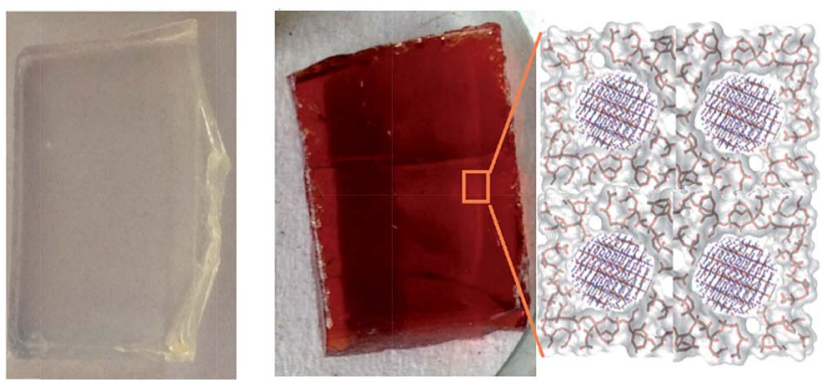

Fig. 1 Images of transparent empty silica monolith (left) and SNPsilica nanocomposite (right).

\subsection{Nitrogen adsorption-desorption analysis}

In order to characterise the porosity of the undoped xerogels, nitrogen sorption isotherms (Fig. $1 \mathrm{~S}(\mathrm{a}) \dagger$ ) were recorded at $77 \mathrm{~K}$ using a Quantachrome Porosimeter Autosorb 1-LP-MP, after an outgassing process of several hours at $150^{\circ} \mathrm{C}$ under a secondary vacuum. The specific surface area was determined by the Brunauer-Emmett-Teller (BET) method. ${ }^{26}$ The total pore volume was determined at $P / P_{0}=0.99938$. The pore size diameter and distribution were calculated according to the Barrett-JoynerHalenda (BJH) ${ }^{27}$ model. As previously reported for this type of xerogel, ${ }^{25}$ the specific surface area and the total pore volume are estimated to $360 \mathrm{~m}^{2} \mathrm{~g}^{-1}$ and $0.49 \mathrm{~cm}^{3} \mathrm{~g}^{-1}$, respectively, ${ }^{26}$ by the BET method. ${ }^{26}$ The pore size distribution, derived by the BJH method, ${ }^{27}$ is centered around $5.8 \mathrm{~nm}$, with a narrow size distribution (Fig. 1S(b)†). More details on the nitrogen adsorption desorption analysis are given in the ESI. $\dagger$

\subsection{Total scattering $X$-ray measurements and PDF analysis}

The total scattering X-ray measurements were performed at the High Energy Scattering Beamline ID15B of the European Synchrotron Radiation Facility (ESRF). The data-sets were collected using a Perkin Elmer flat panel detector and a high energy monochromatic beam ( $56 \mathrm{keV}, \lambda=0.22299 \AA)$. Such an experimental set-up allows recording the total scattering patterns for the unloaded and loaded monoliths up to a high momentum transfer $\left(Q \sim 24 \AA^{-1}\right)$, which is crucial for providing high and adequate real-space resolution of the PDF peaks for the quantitative structural analysis. The collected data were then corrected for experimental effects, and the differential experimental atomic pair distribution function (d-PDF) was obtained following the procedure we reported earlier. ${ }^{18}$ All data processing was done using Fit-2 $\mathrm{D}^{28}$ and Gudrun $\mathrm{X}^{29}$ software.

\subsection{Solid-state NMR spectroscopy}

All the experiments were carried out on a high-field Bruker NMR Avance III spectrometer operating at $14 \mathrm{~T}\left({ }^{1} \mathrm{H}\right.$ NMR frequency of $600 \mathrm{MHz}$ ) with a Bruker $4 \mathrm{~mm}$ MAS triple-resonance probe. The MAS spinning speed was set to $12.5 \mathrm{kHz}$.

For the ${ }^{13} \mathrm{C}$ MAS experiments, the rf-field strength applied for the ${ }^{13} \mathrm{C}$ pulse was set to $46 \mathrm{kHz}$, leading to a $90^{\circ}$ pulse length of $5.4 \mu$ s. During acquisition, SPINAL-64 (ref. 30) heteronuclear decoupling was applied at an rf-field strength of $80 \mathrm{kHz}$. For the hybrid SNP@SiO ${ }_{2}$ sample, the interscan delay (rd) was set to $5 \mathrm{~s}$ and $84 \mathrm{~s}$, and 1600 scans were completed in a total experimental time of about $2 \mathrm{~h}$ and $37 \mathrm{~h}$, respectively.

For the ${ }^{23} \mathrm{Na}$ MAS experiments, the rf-field strength applied for the ${ }^{23} \mathrm{Na}$ pulse was set to $50 \mathrm{kHz}$, with an excitation pulse length of $1 \mu \mathrm{s}$ ( $18^{\circ}$ flip angle). During acquisition, SPINAL-64 (ref. 30) heteronuclear decoupling was applied at an rf-field strength of $80 \mathrm{kHz}$. The interscan delay was set to $5 \mathrm{~s} ; 128$ scans were completed in a total experimental time of about $10 \mathrm{~min}$.

For the hydration study (Fig. 8), the open NMR rotors were stored overnight with saturated solutions of $\mathrm{LiBr}, \mathrm{CaCl}_{2}$ and $\mathrm{KCl}$ salts stabilising the atmosphere at $6 \%, 29 \%$ and $85 \%$ relative humidity $\left(25{ }^{\circ} \mathrm{C}\right)$, respectively. For all the experiments, the temperature at the stator output was stabilised at $298 \mathrm{~K}$.

The Dmfit ${ }^{31}$ program was used for spectral deconvolution, as well as for fitting the ${ }^{13} \mathrm{C}$ chemical shift anisotropy and second order quadrupolar effects on the line shapes of the ${ }^{23} \mathrm{Na}$ central peaks. All the spectra were processed with the GSim program developed by V. Zorin. ${ }^{32}$

\section{Results and discussion}

\subsection{Experimental differential PDF (d-PDF)}

The total scattering patterns of the empty and the loaded monoliths are given in Fig. 2a. Both patterns exhibit the typical amorphous silica features as also observed for the SNP
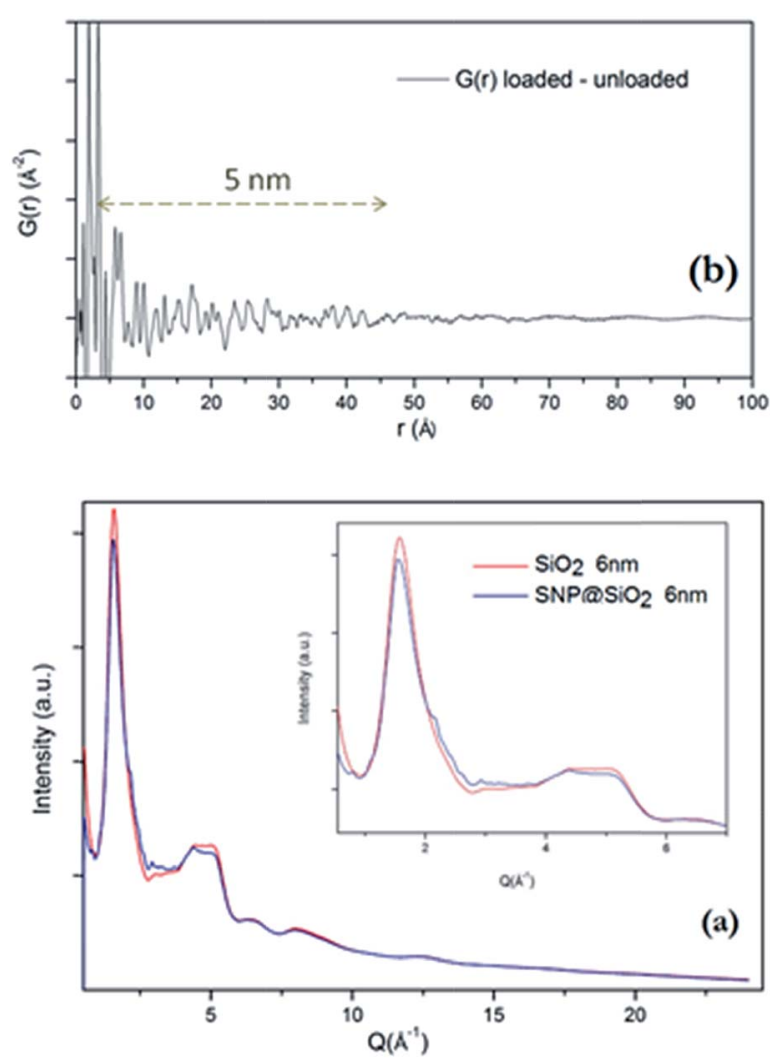

Fig. 2 (a) The total scattering patterns of unloaded (red) and loaded (blue) monoliths. (b) The corresponding differential-PDF. 
complexes incorporated in silica monoliths with pore sizes in the range of $1-3 \mathrm{~nm} .{ }^{18}$ Besides the main peak at $Q \sim 1.64 \AA^{-1}$, one can also observe additional broad peaks in the $Q$-range of 2.5-4 $\AA^{-1}$ in the loaded monolith, indicating the formation of a nanocrystalline SNP phase inside the silica monolith. The resulting differential atomic d-PDF is shown in Fig. 2b. For the subsequent analysis and discussion of the results, we divided this experimental d-PDF into three regions: the short-range (interatomic distances below $\sim 8 \AA$ ), the medium-range $(8-50$ $\AA)$ and the long-range ( $>50 \AA)$ region. The short-range region gives information on the first few coordination spheres and thus mainly contains the information about the intramolecular SNP structure and the nearest neighbours in the crystal, such as the cations. The medium and long ranges contain information on the periodicity of the synthesised particles and on their size. From the d-PDF diagram, we can clearly observe well-defined features up to about $50 \AA$, which reflects the crystalline character of the SNP within the SNP-silica nanocomposites. The rapid fall-off of d-PDF oscillations beyond $r \sim 50 \AA$ indicates that the range of the structural coherence is limited to $\sim 5 \mathrm{~nm}$. Indeed, the finite crystallite size diminishes the number of atom pairs separated by distances larger than the crystallite diameter and, consequently, leads to abrupt damping PDF features. We therefore refer, as a first approximation, to the confined SNP particles as $5 \mathrm{~nm}$ in diameter. In order to obtain the structural model corresponding to the differential PDF, we performed the following modelling strategy by using the DISCUS program. ${ }^{33}$ At first, the d-PDF pattern was fitted by simulating a spherical SNP crystal with a diameter of $5 \mathrm{~nm}$. This model was constructed on the basis of the molecular structure of the bulk crystalline material at room temperature (297 K).

The spherical shape was obtained by applying a spherical envelope function of $5 \mathrm{~nm}$ diameter; in other words, a spherical nanoparticle was cut out of the infinite perfect crystal. One should note that the spherical envelope function gives the best fit of the calculated PDF to the experimental data, compared to the anisotropic envelope functions, like cylinders, that we tested. The resulting simulated d-PDF pattern is shown in Fig. 3a in comparison with the experimental d-PDF. We observe that this model does not describe well the short-range region; in particular, the amplitudes are largely underestimated. These residual sharp peaks at low $r$ values might be due to the presence of a second contribution (phase) confined in the porous environment. This will be discussed in detail in the next paragraph. The medium-range region, on the other hand, is rather well described, although the oscillations damp off too fast with respect to the experiment (see the inset in Fig. 3a). Since the damping is directly related to the particle size, we refined the nanoparticle size (i.e. the diameter of the envelope function) using the experimental data in the range of 8-70 $\AA$; thereby, excluding the short-range region which should bias the result. This refinement provided an improved fit and an average particle size of about $6 \mathrm{~nm}$ (Fig. 3b). This result is in good agreement with the pore size distribution obtained from the nitrogen sorption measurements (see ESI $\dagger$ ).

Now with a good description for the medium- and long-range regions, we return to the residual sharp peaks observed in the
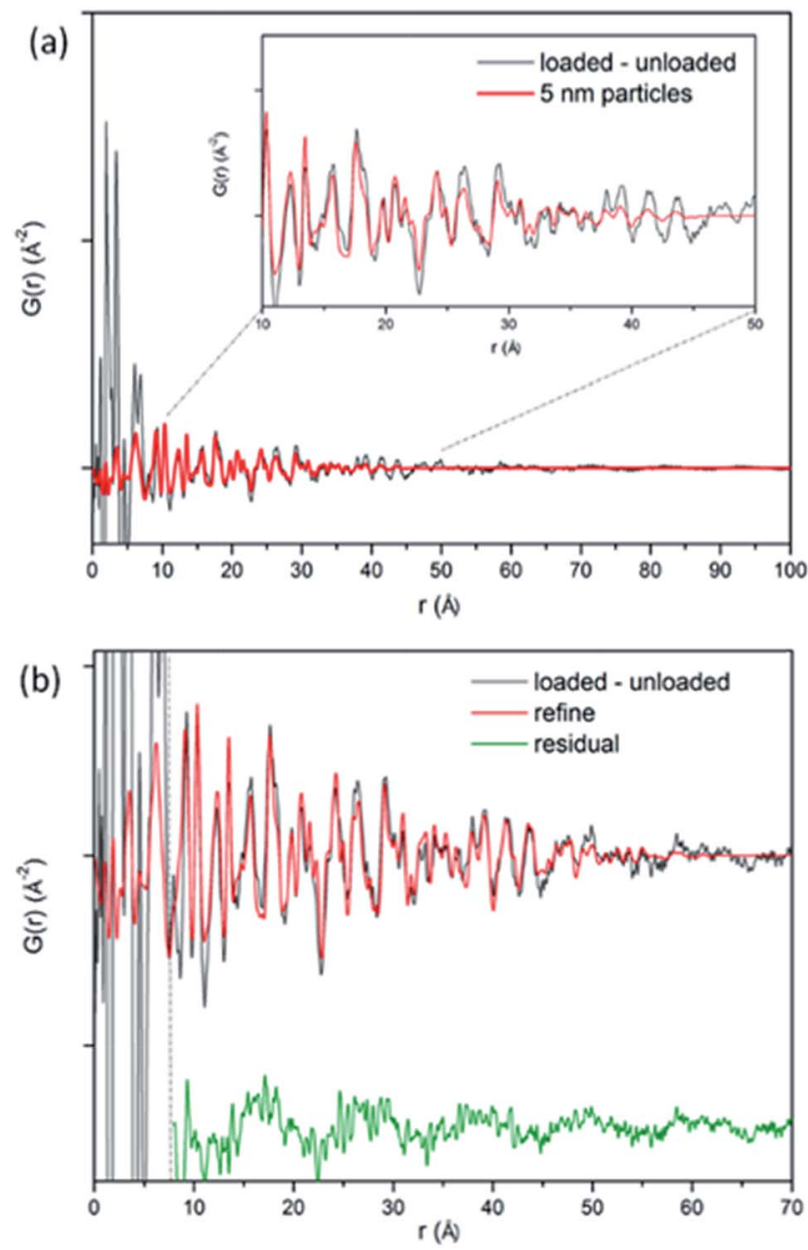

Fig. 3 (a) The experimental d-PDF (black) fitted with a $5 \mathrm{~nm}$ spherical particle model based on the bulk crystalline structure (red). (b) A similar fit with refinement of the nanoparticle size using the experimental data in the range of $8-70 \AA$.

short-range region $(r<8 \AA)$. In order to determine the structural model corresponding to these residual peaks, we subtracted the modelled PDF contribution of the $6 \mathrm{~nm}$ crystalline particles from the experimental d-PDFs pattern, as shown in Fig. 4. The resulting difference exhibits a close resemblance with the PDF modelled with isolated SNP molecules, ${ }^{18}$ indicating that the additional contribution is due to the presence of isolated SNP molecules distributed in the porous network (see the inset in Fig. 4).

\subsection{Solid-state NMR approach}

Following the strategy we established in our last study of an SNP inside a $1 \mathrm{~nm}$ pore size matrix, ${ }^{18}$ we performed a complementary multinuclear MAS-NMR study. The purpose was: (i) to confirm the structural features obtained from the PDF analysis, and (ii) to characterise the physical state of the confined SNP complexes depending on their hydration levels.

As a first step, we validate the differential PDF approach by comparing the ${ }^{29} \mathrm{Si}$ spectra of the empty and the loaded matrices (spectra not shown). Indeed, as in our last study, we 


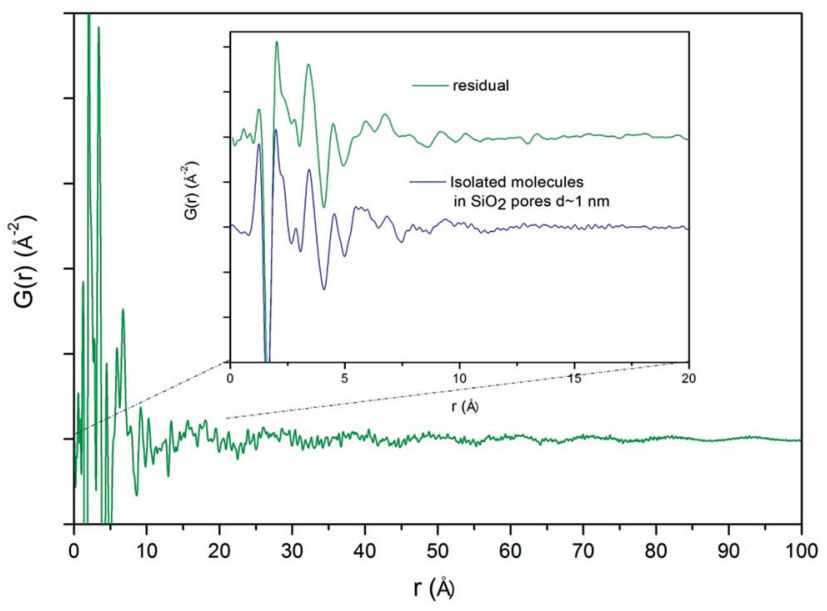

Fig. 4 The d-PDF pattern obtained after the subtraction of the $6 \mathrm{~nm}$ nanocrystalline particles' contribution from the experimental d-PDF. The inset shows the comparison between the resulting $d$-PDF (in green) and the PDF of the isolated SNP molecules from ref. 18.

verified that the spectrum of the loaded matrix did not show any additional chemical sites, nor an increase in the line widths. This indicates that no modification of the matrix is induced by the SNP embedding procedure.

The second step corresponds to the characterisation of the SNP guest complex. For this purpose, two NMR active nuclei were chosen: ${ }^{13} \mathrm{C}$ NMR, to focus on the $\left[\mathrm{Fe}(\mathrm{CN})_{5} \mathrm{NO}\right]^{2-}$ anions and ${ }^{23} \mathrm{Na} \mathrm{NMR}$, to probe the Na counter-ions.

The ${ }^{13} \mathrm{C}$ MAS-NMR spectra present very different features depending on the acquisition parameters, especially when the

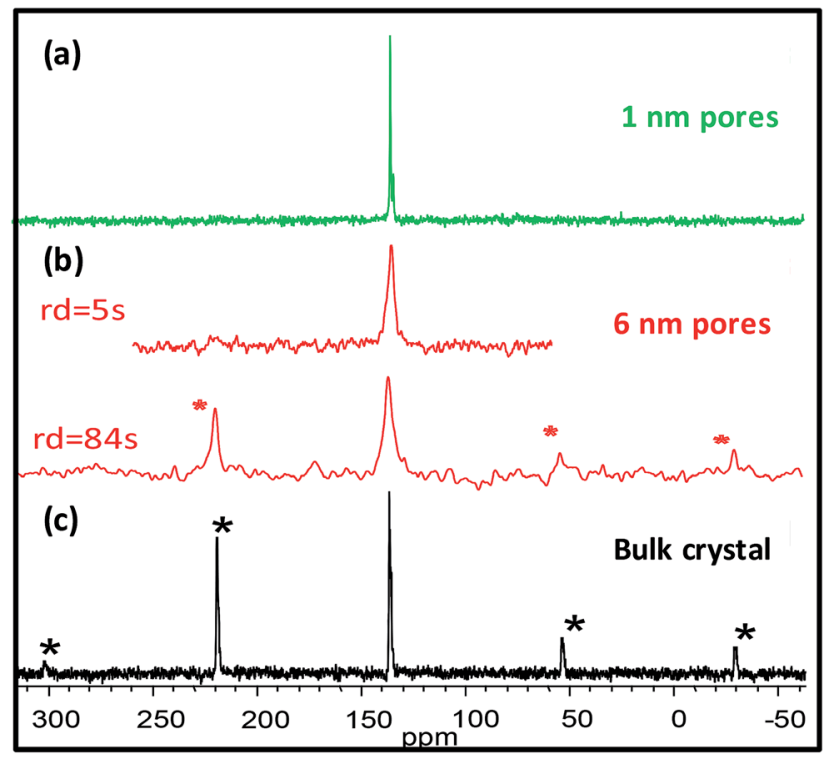

Fig. $5{ }^{13} \mathrm{C}$ MAS NMR spectra $\left(\nu^{\mathrm{MAS}}=12.5 \mathrm{kHz}\right)$ of the SNP recorded in different environments: (a) loaded into a $1 \mathrm{~nm}$ pore $\mathrm{SiO}_{2}$ matrix from ref. 18, (b) loaded into a $6 \mathrm{~nm}$ pore $\mathrm{SiO}_{2}$ matrix, and (c) inside the bulk crystalline material. For (b), the spectra have been recorded with recycling interscan delays of $5 \mathrm{~s}$ (top) and $84 \mathrm{~s}$ (bottom), respectively. The spinning sidebands are marked by asterisks. interscan recycling delay is varied (Fig. 5). For shorter recycling interscan delays (e.g. $5 \mathrm{~s})$, the detected isotropic ${ }^{13} \mathrm{C}$ signal is centred on the chemical shifts in the solution state ( $\sim 134 \mathrm{ppm})$. No MAS sidebands reflecting the large $\mathrm{Fe}^{13} \mathrm{C} \equiv \mathrm{N}$ chemical shift anisotropy $\left(\delta^{\text {csa }}=208 \mathrm{ppm}\right)$ are observed. This recalls the results obtained for the SNP confined in the $1 \mathrm{~nm}$ pore size matrix, and shows evidence for sub- $\mu$ s isotropic rotational diffusion and indicates the presence of a 'liquid like' state through solvation by free water molecules. ${ }^{18}$ When longer recycling delays are used (e.g. $84 \mathrm{~s})$, the detected isotropic ${ }^{13} \mathrm{C}$ signal shifts towards the bulk crystalline values ( 136 ppm) and MAS sidebands appear. These sidebands, marked by asterisks in Fig. 5 , reflect the strong $\mathrm{Fe}^{13} \mathrm{C} \equiv \mathrm{N}$ chemical shift anisotropy and can only appear if the phase is rigid with an accordingly longer $T_{1}$ relaxation time. Therefore, these spectral differences for short and long relaxation delays, respectively, reveal the coexistence of a mobile and a rigid phase of SNP.

Despite the fact that these results clearly confirm the presence of two different SNP phases, the poor ${ }^{13} \mathrm{C}$ sensitivity prevents a precise quantification of the ratio between the phases. The large line-broadening consequently applied for the processing (i.e. $\sim 300 \mathrm{~Hz}$ ) also limits the spectral resolution of the central line. It is therefore not possible, from the ${ }^{13} \mathrm{C}$ spectra alone, to characterise the degree of order in the rigid phase and to distinguish between the amorphous and a crystalline structure of the rigid phase. Nevertheless, since the longitudinal relaxation times of the phases differ by about two orders of magnitudes $\left(T_{1}=300 \mathrm{~ms} / 37 \mathrm{~s}\right.$ for the mobile/rigid phases, respectively), a bi-exponential fitting of a saturation recovery experiment can provide an estimation of the relative ratio of the two phases. This analysis shows that less than $35 \%$ of the SNP molecules are in a mobile phase.

The ${ }^{23} \mathrm{Na}$ MAS-NMR spectra also present evidence for the coexistence of a rigid and a 'liquid like' phase, and the good sensitivity of the nuclei permits the structural characterisation of the rigid phase (Fig. 6). The rigid phase component of the spectrum shows two distinct sites: $\mathrm{Na}_{1}\left(\delta_{1}^{\text {iso }}=-5.7 \mathrm{ppm}\right)$ and $\mathrm{Na}_{2}\left(\delta_{2}^{\text {iso }}=-1.9 \mathrm{ppm}\right)$. The first site has a quadrupolar constant, $C_{\mathrm{Q} 1}=1369 \mathrm{kHz}$, and an asymmetry of $\eta_{1}=0.9$, as illustrated by the characteristic central line-shape, while the second site $\mathrm{Na}_{2}$ has a smaller constant $C_{\mathrm{Q} 2}=615 \mathrm{kHz}$ and $\eta_{2}=0.3$ and exhibits visible quadrupolar satellites.

The chemical shifts and quadrupolar constants are the same as for the ${ }^{23} \mathrm{Na}$ nuclei inside the bulk crystalline SNP structure; therefore, we can conclude that the rigid SNP phase in the nanocomposite is crystalline, and has an identical structure as in the bulk crystal. Given the high sensitivity of the ${ }^{23} \mathrm{Na}$ nuclei, it is relatively easy to deconvolute the central resonance lines in order to extract the relative ratio between the crystalline and mobile phases (Fig. 7). Taking into account the ${ }^{23} \mathrm{Na}$ magnetisation distributed in the satellite transitions, we find that, for the sample studied, $25 \%$ of the SNP complexes are in a 'liquid like' state.

Since we know from our previous study that water molecules can easily exchange between the matrix pores and the atmosphere, we also decided to study the effect of hydration on the confined nanoparticles. Fig. 8 presents the ${ }^{23} \mathrm{Na}$ MAS-NMR 


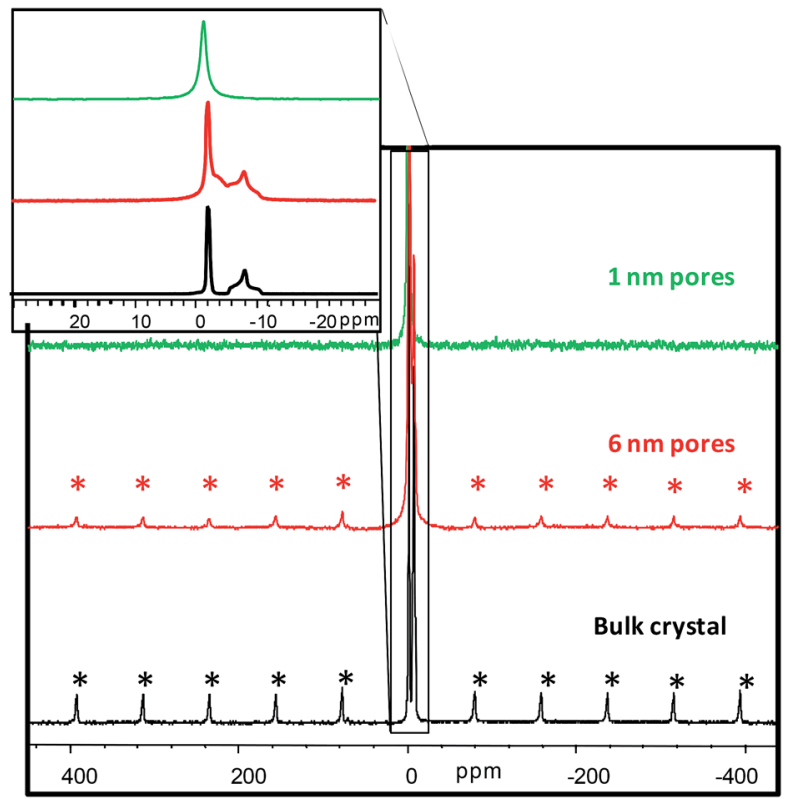

Fig. $6{ }^{23} \mathrm{Na}$ MAS NMR spectra $\left(\nu^{\mathrm{MAS}}=12.5 \mathrm{kHz}\right)$ of the SNP recorded in different environments: (a) loaded into a $1 \mathrm{~nm}$ pore $\mathrm{SiO}_{2}$ matrix from ref. 18, (b) loaded into a $6 \mathrm{~nm}$ pore $\mathrm{SiO}_{2}$ matrix, and (c) inside the bulk crystalline material. The MAS satellite sidebands are marked by asterisks. The inset focuses on the centre band of the spectra.

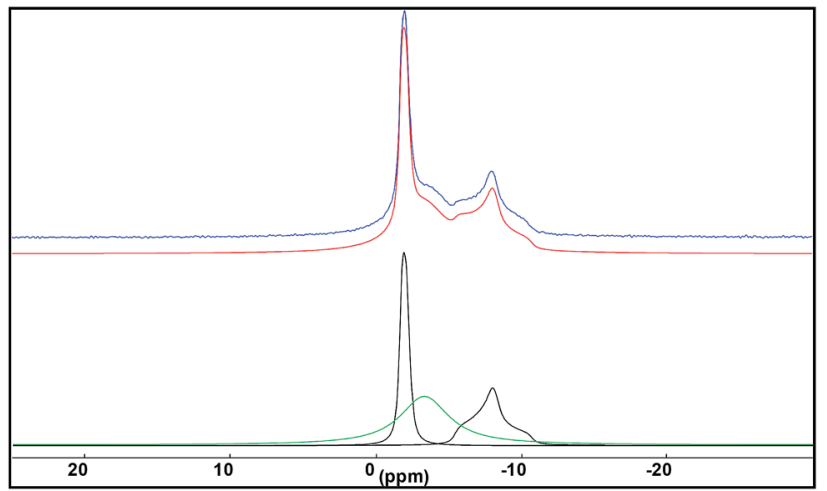

Fig. $7{ }^{23} \mathrm{Na}$ MAS NMR spectra $\left(\nu^{\mathrm{MAS}}=12.5 \mathrm{kHz}\right)$ of the SNP loaded into a $6 \mathrm{~nm}$ pore size $\mathrm{SiO}_{2}$ matrix. The top spectrum corresponds to the experiment and the middle spectrum to a model based on the sum of the fitted components. The bottom spectra show an overlap of the fitted components.

spectra for samples stored in three different environments of $6 \%, 29 \%$ and $84 \%$ relative humidity $(\mathrm{RH})$. The spectral deconvolution permits the monitoring of the effect of hydration on the distinct phases of the confined SNP. An increase in relative humidity affects the solvated phase in a similar way to that observed for $1 \mathrm{~nm}$ pore nanocomposites, ${ }^{18}$ because the signal becomes narrower and shifts towards the aqueous solution reference at $0 \mathrm{ppm}(\mathrm{NaCl} 1 \mathrm{M})$. The frequency range indicates the maintenance of a six-fold coordination of the $\mathrm{Na}^{+}$cations, but the displacement to higher frequencies of this component (in green) reflects a reduction of the average $\mathrm{Na}-\mathrm{O}$ distances. ${ }^{34,35}$

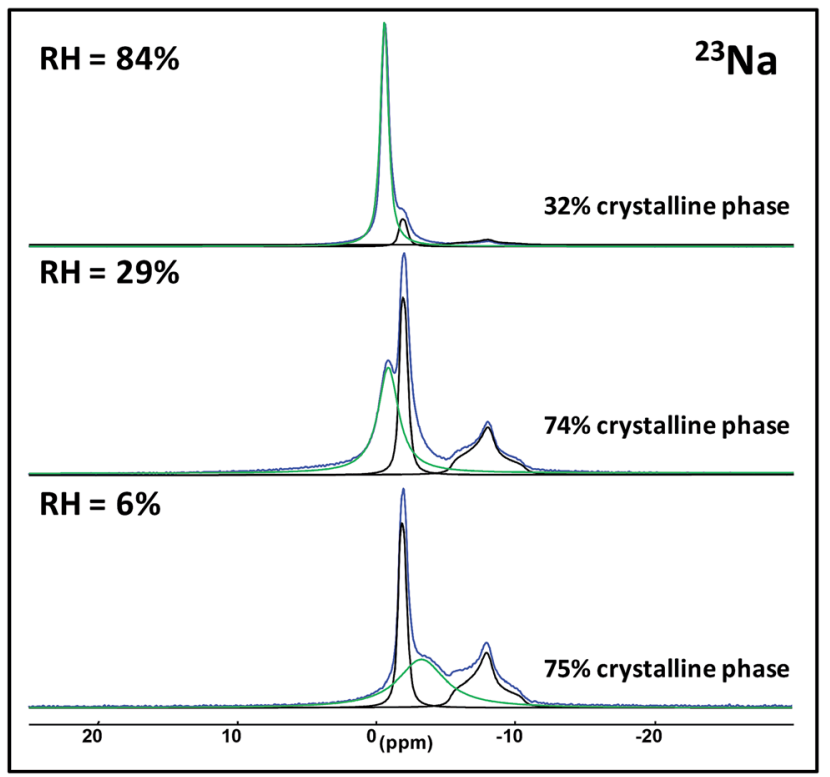

Fig. 8 Effect of hydration on the ${ }^{23} \mathrm{Na}$ MAS NMR spectra $\left(\nu^{\text {MAS }}=12.5\right.$ $\mathrm{kHz}$ ) of the SNP loaded into a $6 \mathrm{~nm}$ pore size $\mathrm{SiO}_{2}$ matrix. The experimental spectra are overlaid with the fitted decomposition. The loaded xerogels have been stored overnight at $84 \%, 29 \%$ and $6 \%$ relative humidity (top to bottom).

Additionally, since the simultaneous line narrowing indicates the increased dynamics of the cations, we can therefore conclude that the increase in relative humidity leads to a better solvation of the cations in the mobile phase, until they are completely surrounded by water molecules. On the contrary, the signals from the crystalline phase are not modified by hydration, which indicates that there is no change in the nanoparticle structure. However, it is very interesting to note that the ratio between the crystalline and solvated complexes is modified by atmospheric humidity. For a relatively dry atmosphere up to $29 \%$ relative humidity, the relative surfaces of the peaks do not change, since the narrowing of the mobile component is compensated by an increased intensity. Therefore, the amount of molecules in the crystalline state does not vary and stays around $75 \%$, which suggests that the remaining $25 \%$ of the SNP complexes are located in a space of the gel matrix that prevents particle crystallisation.

This space could be located at the interface between the nanoparticles and the pore surface or in residual pores of a smaller size. When the humidity increases up to $84 \% \mathrm{RH}$, the amount of $\mathrm{Na}$ atoms in the crystalline state drops down to $32 \%$. This variation between intermediate and high hydration points towards the formation of a liquid-like contact layer of SNP around the pore surface. It also implies that atmospheric humidity can very easily dissolve the nanocrystals inside the pores, a behaviour that is not observed for the bulk macrocrystals.

\subsection{Discussion}

The combination of total X-ray scattering coupled to PDF analysis and solid-state NMR spectroscopy allows for 
characterisation of the nanoscale hybrid materials. In the following section, we discuss the requirements and precautions to be applied to such a study, as well as some caveats for the interpretation of the results.

The structural analysis is based on the experimental differential PDF obtained by direct subtraction of a reference PDF measured for the empty silica matrix from that of the guestloaded framework, therefore eliminating the contributions arising from the host-host atomic correlations (i.e. the interatomic distances), and hence, recovering the structural properties of the guest. This requires that the matrix itself is not significantly affected by impregnation with the guest. This is verified by the ${ }^{29} \mathrm{Si}$-NMR analysis. The differential PDF then contains the structural information (i.e. the distances between the pairs of atoms) of the guest, but also of the possible pairs between the host and guest (e.g. the Fe-Si distances), as these are not cancelled out by the subtraction procedure. In the present study, these distances did not appear distinctly in the $G(r)$, because we are dealing with an amorphous matrix and a randomly oriented guest. This configuration leads to broadsmeared features, since different pairs show slightly different distances. However, we could expect some periodicity, since the nanoparticles themselves are perfectly ordered and the corresponding pairs of distances, especially between $\mathrm{Fe}$ and $\mathrm{Si}$ atoms, should reflect the periodicity of the Fe in the nanoparticles. Inspection of the residuals in Fig. $3 \mathrm{~b}$ indeed reveals such a periodicity of about $12 \AA$, which corresponds to twice the distance of $\mathrm{Fe}-\mathrm{Fe}$ in SNP. An interesting result is that, in one nanopore $6 \mathrm{~nm}$ in diameter, about 113 unit cells of SNP (6 nm particle) were incorporated, and with $Z=4$ (the cell unit formula), only 392 molecules are needed to form nanoparticles of SNP with clearly visible maxima in the diffracted intensity. This example clearly illustrates that the d-PDF approach is very helpful in the study of host-guest systems, since it limits the following modelling to the guest species and thus considerably simplifies the task. In particular, the d-PDF reduces the difficulties due to the overlapping peaks from the host and guest present in the total PDF of the host-guest system. Nevertheless, as for all PDF measurement, high resolution and good statistics are mandatory. This approach was also applied successfully to probe the structural information of a wide variety of host-guest materials, including other coordination framework materials, porous carbons, Prussian Blue analogues and light element nanocomposites. ${ }^{36-38}$ Since NMR provides chemical resolution, NMR distinction between the host and the guest ions and counter-ions is trivial, due to their different composition (i.e. ${ }^{29} \mathrm{Si},{ }^{13} \mathrm{C},{ }^{23} \mathrm{Na}$ ). Therefore, the limiting factor remains the relatively low sensitivity inherent in the NMR technique. Nevertheless, even for heterogeneous systems, like the one studied here, we could detect the presence of nanocrystalline particles both from the signal of the nitroprusside ions $\left({ }^{13} \mathrm{C}\right)$ and from sodium counter-ions $\left({ }^{23} \mathrm{Na}\right)$, even without isotopical labelling.

Concerning the crystallinity and particle size of the embedded species, the PDF analysis provides very detailed insights. As shown above, it relies only on the structural model of the bulk material and a simple envelope function. This type of analysis is very sensitive to the particle size and form, ${ }^{\mathbf{3 9 , 4 0}}$ because the relative strengths of the $G(r)$ amplitudes depend on the number of pairs present at a defined distance $r$. Additionally, different envelope functions are available in the analysis software to simulate different particle shapes. ${ }^{33,40}$ More difficult is the analysis in the case of a partial crystallinity or if a large distribution of particle size is present. If the deviation from the perfect crystallinity is small, this would be reflected in the width of the $G(r)$ amplitudes. Since this effect is superimposed on other contributions, such as the thermal motion or other kinds of dynamical behaviour, a deconvolution is necessary, but this is non-trivial. In all these cases, the NMR analysis provides essential and complementary structural information, since NMR probes the local structure and rotational dynamics. As demonstrated for our case, the study of counter-ions can be especially suited to answer this question, since both elements of the complex show important differences in the NMR signal between the crystalline, amorphous and liquid states. By evaluating the area of the corresponding NMR lines, it is even possible to quantitatively determine the ratio between the different distinct physical states of the guest. This is essential in the assessment of such materials with respect to stability and its applications, as illustrated in the example of the solubility of SNP as a function of the surrounding humidity level. Even if we suggest that the solvated SNP complexes form a contact layer around the nanocrystals inside the pores, the question of the location of the liquid-like molecules in the matrix is not fully answered. The fast spin lattice relaxation rates $(\sim 1 \mathrm{~ms})$ of the ${ }^{23} \mathrm{Na}$ nuclei, as well as the low sensitivity of the ${ }^{13} \mathrm{C}$ nuclei, prevent the use of $2 \mathrm{D}$ chemical exchange NMR experiments, which should be able to address this point. Future work on ${ }^{13} \mathrm{C}$, ${ }^{15} \mathrm{~N}$ isotopically labelled composites studied at variable temperatures should bring about more quantitative insights into the dynamics of the confined complexes.

\section{Conclusions}

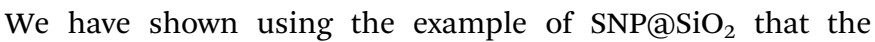
combination of NMR and total X-ray scattering coupled to PDF analysis allows for a detailed structural description of the embedded guest in a porous host. We found that 'bulk-like' crystalline nanoparticles of SNP can be grown inside porous $\mathrm{SiO}_{2}$ matrices with a pore diameter as small as $6 \mathrm{~nm}$. This result is quite exceptional, since the pore diameter is only about ten times larger than the complex size. We have also shown that the average nanoparticle size is about $6 \mathrm{~nm}$, which means that they almost completely fill the matrix pores. Nevertheless, we found that this crystallinity is still partial, since two distinct physical states of SNP are detected. Since crystalline nanoparticles and solvated complexes of SNP could be clearly distinguished, their evolution could thus be investigated as a function of the external parameters. We applied this method to study the stability of the SNP host, a prototype of an API, and could show that as a function of atmospheric relative humidity, the ratio between the 'liquid-like' and nanocrystalline SNP changes. These dissolution properties were not observed for bulk SNP exposed to identical conditions. Therefore, this might be useful 
for the pharmacological application of SNP, since as a function of particle size, the solubility rate can be tailored and it can thus be adapted to the purpose-defined bioavailability. In any case, this method can also be used to study crystallisation behaviour inside functional materials and to learn more about the thermodynamics in nano-sized systems, as well as about the early stages of crystallisation.

\section{Acknowledgements}

This work was supported by the Universite de Lorraine, the CNRS and the Institut Jean Barriol which are gratefully acknowledged. T. W. is very grateful for his stay as invited professor at the CRM2, Université de Lorraine. The authors from the PhLAM thank the "Fonds Européen de Développement Economique Régional" and the Labex CEMPI and Equipex FLUX through the "Programme Investissements d'Avenir".

\section{Notes and references}

1 C. Barbé, J. Bartlett, L. Kong, K. Finnie, H. Q. Lin, M. Larkin, S. Calleja, A. Bush and G. Calleja, Adv. Mater., 2004, 16, 19591966.

2 J. Li, L. Xu, H. Liu, Y. Wang, Q. Wang, H. Chen, W. Pan and S. Li, Int. J. Pharm., 2014, 467, 9-18.

3 C. Argyo, V. Weiss, C. Bräuchle and T. Bein, Chem. Mater, 2014, 26, 435-451.

4 H. Yan, C. Teh, S. Sreejith, L. Zhu, A. Kwok, W. Fang, X. Ma, K. T. Nguyen, V. Korzh and Y. Zhao, Angew. Chem., Int. Ed., 2012, 51, 8373-8377.

5 D. A. Riccio and M. H. Schoenfisch, Chem. Soc. Rev., 2012, 41, 3731-3741.

6 E. Gedat, A. Schreiber, J. Albrecht, T. Emmler, I. Shenderovich, G. H. Findenegg, H.-H. Limbach and G. Buntkowsky, J. Phys. Chem. B, 2002, 106, 1977-1984.

7 A. Vyalikh, T. Emmler, I. Shenderovich, Y. Zeng, G. H. Findenegg and G. Buntkowsky, Phys. Chem. Chem. Phys., 2007, 9, 2249-2257.

8 T. Azaïs, C. Tourné-Péteilh, F. Aussenac, N. Baccile, C. Coelho, J.-M. Devoisselle and F. Babonneau, Chem. Mater., 2006, 18, 6382-6390.

9 G. T. Rengarajan, D. Enke and M. Beiner, Open Phys. Chem. J., 2007, 1, 18-24.

10 T. Azais, G. Hartmeyer, S. Quignard, G. Laurent and F. Babonneau, J. Phys. Chem. C, 2010, 114, 8884-8891.

11 R. Radhakrishnan, K. E. Gubbins and M. SliwinskaBartkowiak, J. Chem. Phys., 2000, 112, 11048-11057.

12 C. Alba-Simionesco, B. Coasne, G. Dosseh, G. Dudziak, K. E. Gubbins, R. Radhakrishnan and M. SliwinskaBartkowiak, J. Phys.: Condens. Matter, 2006, 18, R15.

13 J. Warnock, D. D. Awschalom and M. W. Shafer, Phys. Rev. Lett., 1986, 57, 1753-1756.

14 R. Evans and U. M. B. Marconi, J. Chem. Phys., 1987, 86, 7138-7148.

15 M. Sliwinska-Bartkowiak, G. Dudziak, R. Sikorski, R. Gras, R. Radhakrishnan and K. E. Gubbins, J. Chem. Phys., 2001, 114, 950-962.
16 J. Czwartos, M. Sliwinska-Bartkowiak, B. Coasne and K. E. Gubbins, Pure Appl. Chem., 2009, 81, 1953-1959.

17 B. Coasne, J. Czwartos, M. Sliwinska-Bartkowiak and K. E. Gubbins, J. Chem. Phys., 2010, 133, 084701.

18 K.-Y. Hsieh, E.-E. Bendeif, A. Gansmuller, S. Pillet, T. Woike and D. Schaniel, RSC Adv., 2013, 3, 26132-26141.

19 A. D. Ivankovich, D. J. Miletich and J. H. Tinker, Int. Anesthesiol. Clin., 1978, 16, 1-29.

20 S. K. Wolfe and J. H. Swinehart, Inorg. Chem., 1975, 14, 10491053.

21 M. Goulkov, D. Schaniel and T. Woike, J. Opt. Soc. Am. B, 2010, 27, 927-932.

22 D. Schaniel, M. Imlau, T. Weisemoeller, T. Woike, K. W. Krämer and H.-U. Güdel, Adv. Mater., 2007, 19, 723726.

23 E. Berrier, C. Zoller, F. Beclin, S. Turrell, M. Bouazaoui and B. Capoen, J. Phys. Chem. B, 2005, 109, 22799-22807.

24 H. El Hamzaoui, R. Bernard, A. Chahadih, F. Chassagneux, L. Bois, D. Jegouso, L. Hay, B. Capoen and M. Bouazaoui, Nanotechnology, 2010, 21, 134002.

25 P. Durand, S. Pillet, E.-E. Bendeif, C. Carteret, M. Bouazaoui, H. El Hamzaoui, B. Capoen, L. Salmon, S. Hébert, J. Ghanbaja, L. Aranda and D. Schaniel, J. Mater. Chem. C., 2013, 1, 1933-1942.

26 S. Brunauer, L. S. Deming, W. E. Deming and E. Teller, J. Am. Chem. Soc., 1940, 62, 1723-1732.

27 E. P. Barrett, L. G. Joyner and P. P. Halenda, J. Am. Chem. Soc., 1951, 73, 373-380.

28 A. P. Hammersley, S. O. Svensson, M. Hanfland, A. N. Fitch and D. Hausermann, High Pressure Res., 1996, 14, 235-248.

29 A. K. Soper and E. R. Barney, J. Appl. Crystallogr., 2011, 44, 714-726.

30 A. Khitrin and B. M. Fung, J. Chem. Phys., 2000, 112, 23922398.

31 D. Massiot, F. Fayon, M. Capron, I. King, S. Le Calvé, B. Alonso, J.-O. Durand, B. Bujoli, Z. Gan and G. Hoatson, Magn. Reson. Chem., 2002, 40, 70-76.

$32 \mathrm{~V}$. Zorin, GSim - visualisation and processing tool for NMR experiments and simulations.

33 R. B. Neder and T. Proffen, Diffuse Scattering and Defect Structure Simulations: A Cook Book Using the Program DISCUS, IUCr Texts on Crystallography, No. 11, Oxford University Press, 2008.

34 X. Xue and J. F. Stebbins, Phys. Chem. Miner., 1993, 20, 297307.

35 Y. Kim and R. James Kirkpatrick, Geochim. Cosmochim. Acta, 1997, 61, 5199-5208.

36 K. W. Chapman, P. J. Chupas and C. J. Kepert, J. Am. Chem. Soc., 2005, 127, 11232-11233.

37 K. W. Chapman, P. J. Chupas, E. R. Maxey and J. W. Richardson, Chem. Commun., 2006, 4013-4015.

38 H. Kim, A. Karkamkar, T. Autrey, P. Chupas and T. Proffen, J. Am. Chem. Soc., 2009, 131, 13749-13755.

39 C. L. Farrow, C.-Y. Ruan and S. J. L. Billinge, Phys. Rev. B: Condens. Matter Mater. Phys., 2010, 81, 134104.

40 K. Page, T. C. Hood, T. Proffen and R. B. Neder, J. Appl. Crystallogr., 2011, 44, 327-336. 